\title{
Effect of saccharin supplementation on weight gain, caloric intake and basal oxygen consumption in Wistar rats
}

\author{
Denise Entrudo Pinto ${ }^{*}$ Kelly C Foletto, Pedro Dal Lago, Ramiro Barcos, Marcello Bertoluci \\ From 20th Brazilian Diabetes Society Congress \\ Porto Alegre, Brazil. 11-18 November 2015
}

\section{Background}

The use of non-caloric sweeteners can interfere in the regulation of appetite, promoting greater food intake and weight gain. In previous data, our results showed that animals who consumed yogurt with saccharin and aspartame had a increase in weight compared to the group using sucrose. However, as the total calorie intake was similar between the groups, we speculated that weight gain might be associated with decreased energy expenditure induced by artificial sweetener.

\section{Aim}

Determine the caloric expenditure at rest in rats receiving saccharin or sucrose for 12 weeks.

\section{Materials and methods}

We conducted a controlled experiment with adult male Wistar rats randomly divided into 3 groups: non-caloric sweetener (saccharin-SAC); calorie sweetener (sucroseSUC) or control ( non sweetened yogurt - CON) given daily over a period of 12 weeks with free chow and water. Weight gain, food intake and water control were determined weekly, basal oxygen consumption was measured at 0,5 and 12 weeks. We used one-way ANOVA with Dunnett's test and ANOVA by repeated measures and mixed model assessment.

\section{Results}

The SAC group promoted greater weight gain than control $(\mathrm{p}=0.031)$. All groups had similar total caloric intake. The maximal oxygen consumption was not diferent between groups during the whole experiment, respectivelly: SAC \footnotetext{
Biomed Central
}

* Correspondence: denise.entrudopinto@icloud.com Universidade Federal do Rio Grande do Sul, Porto Alegre, Brazil (basal $27.72 \pm 1.91 ; 5$ weeks $28.39 \pm 1.96$ and 12 weeks 27.16 \pm 0.87 ), SUC (basal 28.66 \pm 1.96 ; 5 weeks $29.35 \pm 3.16$ and 12 weeks 29.08 \pm 1.61 ) and CONT (basal 27.16 \pm 0.87 ; 6 weeks $28.15 \pm 2.53$ and 12 weeks $27.58 \pm 0.97$ ).

\section{Conclusion}

The cumulative weight gain in the animals fed with saccharin can not be attributed to a reduction in energy

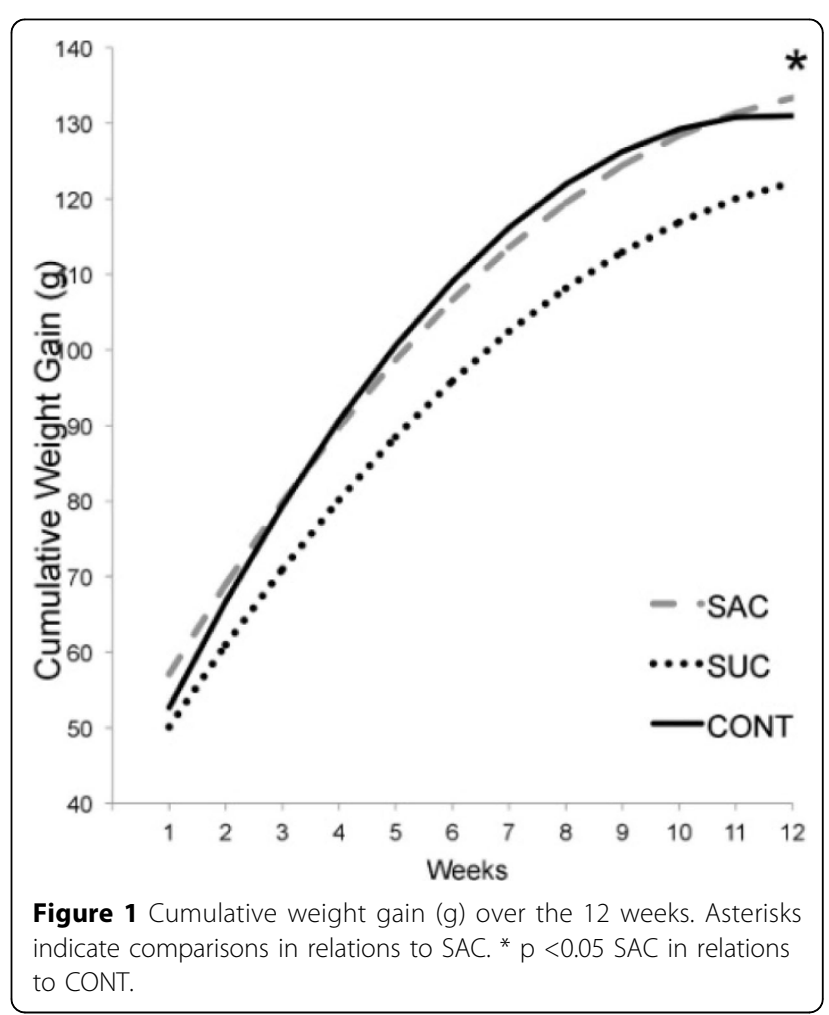

(c) 2015 Pinto et al. This is an Open Access article distributed under the terms of the Creative Commons Attribution License (http:// creativecommons.org/licenses/by/4.0), which permits unrestricted use, distribution, and reproduction in any medium, provided the original work is properly cited. The Creative Commons Public Domain Dedication waiver (http://creativecommons.org/publicdomain/ zero/1.0/) applies to the data made available in this article, unless otherwise stated. 


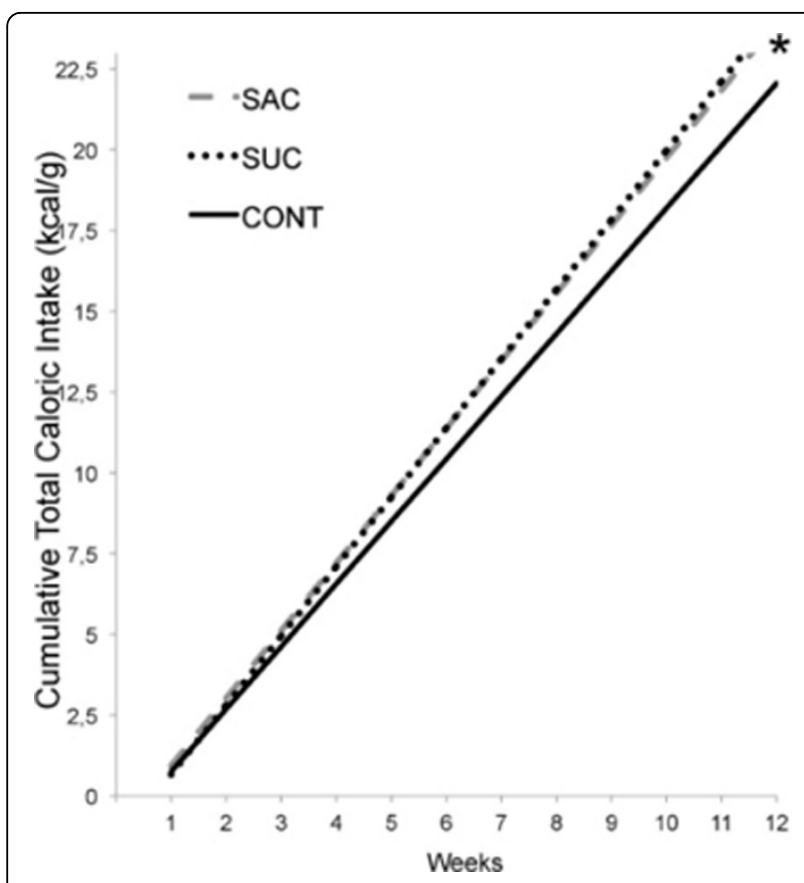

Figure 2 Cumulative total caloric intake connected by the weight weekly $(\mathrm{kcal} / \mathrm{g})$. Asterisks indicate comparisons in relations to SAC. * $p<0.05$ SAC in relations to CONT.

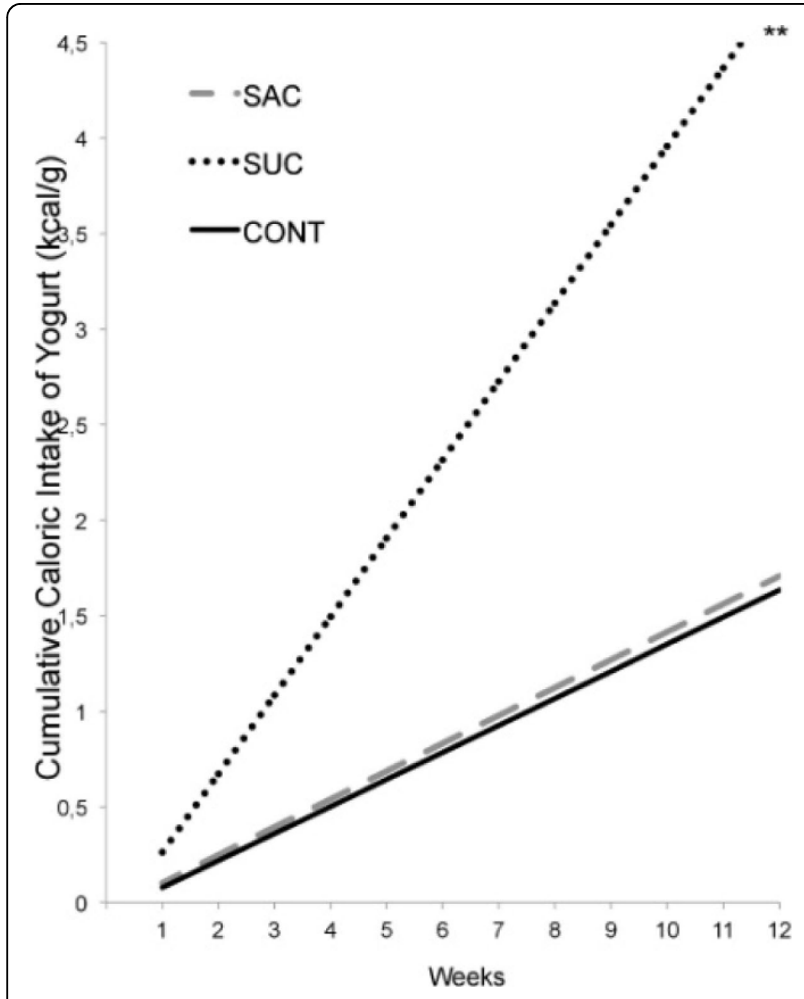

Figure 3 Cumulative caloric intake of yogurt connected by the weight weekly $(\mathrm{kcal} / \mathrm{g})$. Asterisks indicate comparisons in relation to SAC. ${ }^{* *}, p<0.001$ SAC vS SUC.

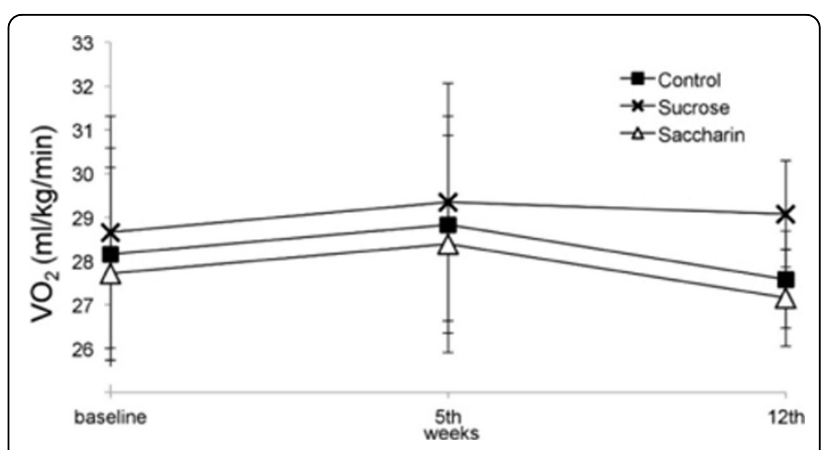

Figure $4 \mathrm{VO}_{2}$ conducted on baseline, $5^{\text {th }} \mathrm{Wk}$ and $12^{\text {th }}$ wk. Analysed by ANOVA of repeated measures.

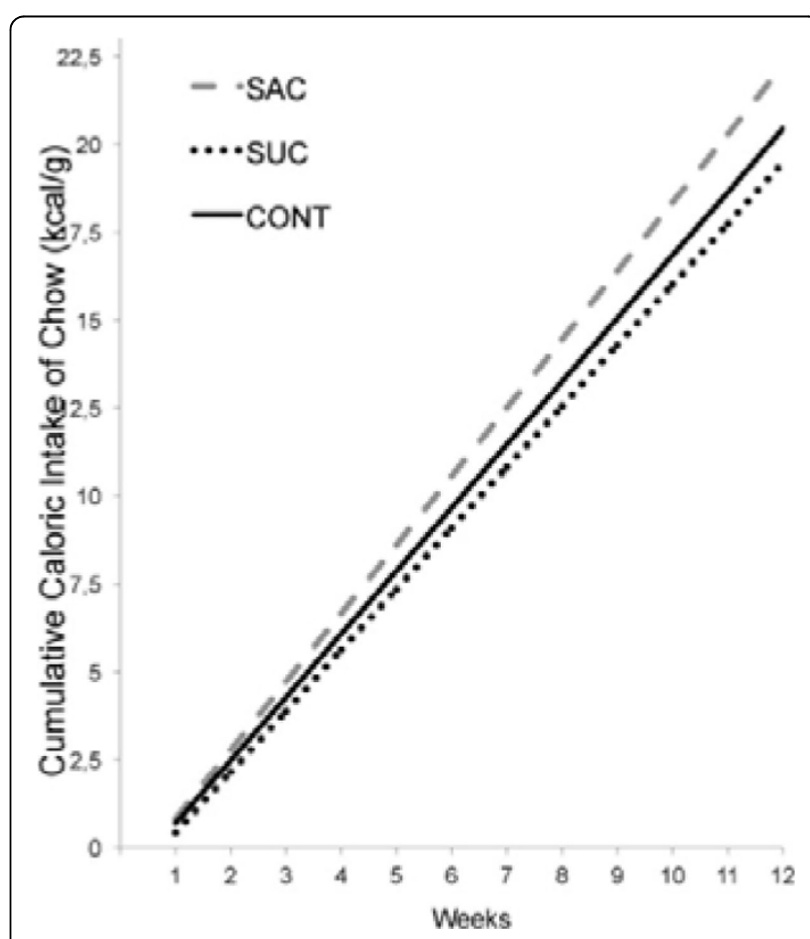

Figure 5 Cumulative caloric intake of chow connected by the weight weekly $(\mathrm{kcal} / \mathrm{g})$. Asterisks indicate comparisons in relation to SAC ${ }^{*} p<0.05$.

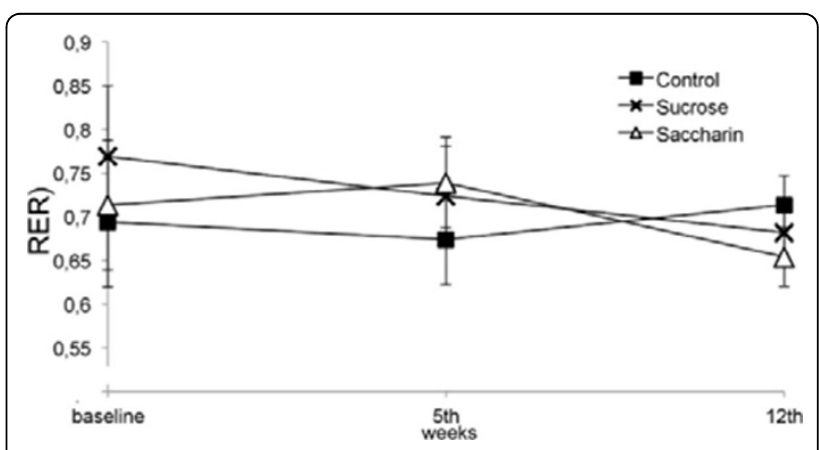

Figure 6 RER (respiratory exchange ratio) conducted on baseline, $5^{\text {th }}$ wk and $12^{\text {th }}$ wk. Analysed by ANOVA of repeated mesures. 


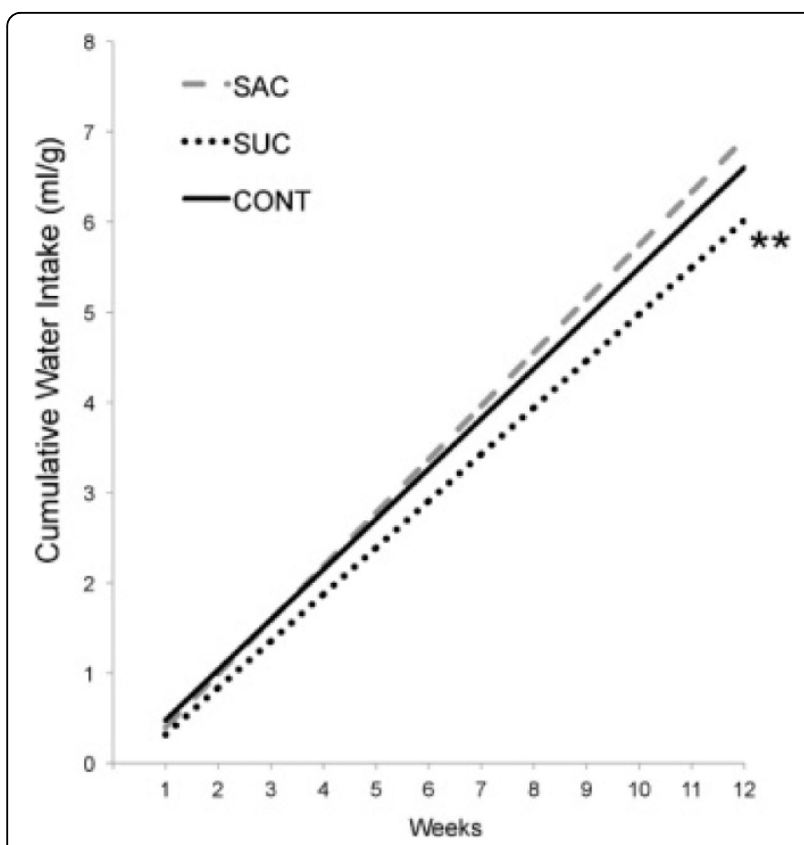

Figure 7 Cumulative water intake connected by the wright weekly $(\mathrm{ml} / \mathrm{g})$. Asterisks indicate comparisons in relation to SAC ** $p<$ 0.005 SAC in relation to SUC.

\begin{tabular}{|c|c|c|c|c|}
\hline & $\begin{array}{c}\text { SAC } \\
(n=12)\end{array}$ & $\underset{(n=10)}{\text { suc }}$ & $\begin{array}{l}\text { CONT } \\
(n=12)\end{array}$ & $p$ value \\
\hline Yogurt intake (\%) & $76.10 \pm 1.41$ & $74.35 \pm 2.18$ & $73.26 \pm 1.81$ & $\rho=.52$. \\
\hline Basal Weight (a) & $207.33 \pm 9.48$ & $219.00 \pm 6.74$ & $219.67 \pm 8.53$ & $p=.51$ \\
\hline Total Weight Gain (g) & $135.92 \pm 8.89$ & $117.00 \pm 7.05$ & $131.00=7.51$ & $\rho=.26$ \\
\hline $\begin{array}{l}\text { Mean Total Caloric Intake } \\
\text { (kcalg/wk) }\end{array}$ & $2.02 \pm 0.06$ & $2.08 \pm 0.06$ & $1.87 \pm 0.04$ & $p=.03$ \\
\hline $\begin{array}{l}\text { Mean Calories from } \\
\text { Yogurt (kcalg/wik) }\end{array}$ & $0.14 \pm 0.003$ & $\begin{array}{l}0.40 \pm \\
0.01 \cdots\end{array}$ & $0.14 \pm 0.01$ & $p<.001$ \\
\hline $\begin{array}{l}\text { Mean Calories from Chow } \\
\text { (kcalg/wk) }\end{array}$ & $1.88 \pm 0.06$ & $1.68 \pm 0.05^{\circ}$ & $1.73 \pm 0.04$ & $p=.03$ \\
\hline $\begin{array}{l}\text { Mean Water intake } \\
(\mathrm{m} / \mathrm{g} / \mathrm{wk})\end{array}$ & $0.57 \pm 0.01$ & $0.50 \pm 0.02^{*}$ & $0.54 \pm 0.02$ & $p=.02$ \\
\hline \multicolumn{5}{|c|}{$\begin{array}{l}\text { Data are mean } \pm \text { SE. Analysis by ANOVA with Dunnett test. Asterisks indicat } \\
\text { comparison with CONT: } \cdots p<0.001 ; \cdots p<0.005 \text { and } " p<0.05 \text {. }\end{array}$} \\
\hline Figure $\mathbf{8}$ Weight, ca & ric and $v$ & r intake & arameters. & \\
\hline
\end{tabular}

expenditure. Further studies are necessary to determine metabolic causes for weight gain induced by saccharin in rats.

\begin{tabular}{|c|c|c|c|c|}
\hline & $\begin{array}{c}\text { SAC } \\
(n=12)\end{array}$ & $\begin{array}{c}\text { suc } \\
\left(n=10^{n}\right)\end{array}$ & $\begin{array}{l}\text { CONT } \\
(n=12)\end{array}$ & Test value \\
\hline $\begin{array}{c}\mathrm{VO}_{2} \text { baseline } \\
\text { (ml/kg/min) }\end{array}$ & $27.72 \pm 1.91$ & $28.66 \pm 3.08$ & $28.15 \pm 2.53$ & $\rho=.97$ \\
\hline $\begin{array}{l}\mathrm{VO}_{2} \text { wk } 5 \\
(\mathrm{~m} / \mathrm{kg} / \mathrm{min})\end{array}$ & $28.39 \pm 1.96$ & $29.35 \div 3.16$ & $28.83 \pm 2.59$ & $p=.97$ \\
\hline $\begin{array}{l}\mathrm{VO}_{2} \text { wk } 12 \\
\text { (mukg/min) }\end{array}$ & $27.16 \pm 0.87$ & $29.08 \pm 1.61$ & $27.58 \pm 0.97$ & $p=.49$ \\
\hline $\mathrm{AUC} \mathrm{VO}_{2}$ & $334.71 \pm 18.80$ & $349.53 \div 30.78$ & $339.91 \div 23.56$ & $p=.91$ \\
\hline $\begin{array}{l}\text { RER baseline } \\
\left(\mathrm{VCO}_{2} \mathrm{NO}_{2}\right)\end{array}$ & $0.63 \pm 0.21$ & $0.74 \pm 0.38$ & $0.64 \div 0.31$ & $p=.69$ \\
\hline $\begin{array}{l}\text { RER wk } 5 \\
\left(\mathrm{VCO}_{2} \mathrm{NO}_{2}\right)\end{array}$ & $0.74 \pm 0.07$ & $0.72+0.04$ & $0.67 \pm 0.03$ & $p=.66$ \\
\hline $\begin{array}{l}\text { RER wk } 12 \\
\left(\mathrm{VCO}_{2} \mathrm{NO}_{2}\right)\end{array}$ & $0.65=0.04$ & $0.68 \div 0.04$ & $0.71 \div 0.03$ & $p=.47$ \\
\hline AUC RER & $8.51 \pm 0.56$ & $8.66 \pm 0.33$ & $8.28 \div 0.36$ & $p=.83$ \\
\hline AUC of OGTT wk 6 & $10665 \pm 284.64$ & $\begin{array}{c}10894.50= \\
321.18\end{array}$ & $10153 \pm 330.94$ & $p=.68$ \\
\hline AUC of OGTT wk 12 & $10665 \div 284.64$ & $\begin{array}{c}10894.50= \\
321.18\end{array}$ & $10153 \div 330.94$ & $p=.13$ \\
\hline \multicolumn{5}{|c|}{ Data are mean $\approx S E$ for Analysis by ANOVA with Dunnett test; of median \pm interquartile. } \\
\hline $\mathrm{D}_{2}$ & and OGTT & meters. & & \\
\hline
\end{tabular}

Published: 11 November 2015

doi:10.1186/1758-5996-7-S1-A158

Cite this article as: Pinto et al:: Effect of saccharin supplementation on weight gain, caloric intake and basal oxygen consumption in Wistar rats.

Diabetology \& Metabolic Syndrome 2015 7(Suppl 1):A158.

\section{Submit your next manuscript to BioMed Central and take full advantage of:}

- Convenient online submission

- Thorough peer review

- No space constraints or color figure charges

- Immediate publication on acceptance

- Inclusion in PubMed, CAS, Scopus and Google Scholar

- Research which is freely available for redistribution

Submit your manuscript at www.biomedcentral.com/submit 